\title{
Regulierung besonders besorgniserregender Stoffe
}

Anhand welcher Kriterien sollten besonders besorgniserregende Stoffe nach Artikel 57, Buchstabe f, der REACH-Verordnung reguliert werden? Diese Frage wurde von Experten im Rahmen eines Workshops in Berlin diskutiert.

E in Schwerpunkt der Europäischen Verordnung über die Registrierung, Bewertung, Zulassung und Beschränkung von Chemikalien („REACH“-Verordnung) liegt auf den sogenannten „besonders besorgniserregenden Substanzen“ („Substances of Very High Concern“, SVHC). Darunter fallen Stoffe, die Krebs auslösen, das Erbgut schädigen oder die Fortpflanzung gefährden können. Darüber hinaus können nach Artikel 57, Buchstabe f, der Verordnung zusätzlich Stoffe mit anderen, „ebenso besorgniserregenden“ Eigenschaften als SVHC identifiziert werden. Die REACH-Verordnung nennt hierfür jedoch keine konkreten Kriterien. Aus diesem Grund hatten die Bundesanstalt für Arbeitsschutz und Arbeitsmedizin (BAuA) und das Bundesinstitut für Risikobewertung (BfR) zu einem eintägigen Workshop nach Berlin geladen. Diskutiert wurden mögliche Kriterien für solche anderen, „ebenso besorgniserregenden“ SVHC-Eigenschaften. Unter dem Titel „REACH Article 57 (f): Non-Endocrine Disrupting Hu- man Health Hazards Leading to SVHC Identification“äußerten sich Experten nationaler und europäischer Behörden, der chemischen Industrie und von Nichtregierungsorganisationen (NGO) dazu, wie Artikel 57, Buchstabe $\mathrm{f}$, in der Praxis angewandt werden sollte.

\section{Einheitliches Konzept}

Die Experten waren sich auf dem Workshop einig, dass ein einheitliches Konzept für den Umgang mit Artikel 57, Buchstabe $f$, hilfreich wäre. So erfüllen nach ihrer Ansicht beispielsweise Stoffe, die bei Kontakt mit den Atemwegen zu Allergien führen können, die Anforderungen von Artikel 57, Buchstabe f, und sollten somit prinzipiell den besonders besorgniserregenden Substanzen zugerechnet werden können. Darüber hinaus sprachen sich die Vertreter von BAuA und BfR in ihren Vorträgen dafür aus, unter bestimmten Bedingungen auch Stoffe als mögliche SVHC-Kandidaten zu betrachten, die zu schweren Organschäden führen können.
Chemikalien, die nur leichte Gesundheitsschäden hervorrufen oder die ihrer Natur nach ohnehin ein effektives Risikomanagement einfordern, wie beispielsweise stark ätzende Stoffe, würden dagegen in der Regel nicht als SVHC betrachtet werden. Im Laufe der Diskussionen auf dem Workshop stellte sich heraus, dass in vielen Punkten zwar noch weiterer Diskussionsbedarf zwischen Behörden und den Stakeholdern aus Industrie und Nichtregierungsorganisationen besteht. Die Auseinandersetzung mit dem Thema zwischen verschiedenen Interessenvertretern ist aber bereits ein erster gelungener Schritt hin zu einem harmonisierten Vorgehen. BAuA und BfR kommen zu dem Schluss, dass die Einigung auf ein einheitlich zu verwendendes Konzept nicht nur zu mehr Transparenz im Umgang mit Artikel 57, Buchstabe f, führt, sondern auch ein effektives Risikomanagement für bisher nicht ausreichend regulierte gefährliche Stoffe ermöglicht.

Weitere Infos: www.baua.de

\section{Diener electronic wächst weiter}

Der Plasmatechnik-Hersteller Diener electronic befindet sich nach eigenen Angaben weiter auf Wachstumskurs. 2011 konnte das Unternehmen mit einem Umsatz von 8,2 Mio. € eine Steigerung von 24 Prozent im Vergleich zum Vorjahr erzielen.

$\mathrm{N}$ achdem das Unternehmen im Jahr 2009 erst ein größeres Firmengebäude bezogen hatte, expandiert es nun weiter und bezieht in Ebhausen ganz in der Nähe der Firmenzentrale ein weiteres 845 Quadratmeter großes Gebäude. Dort zieht die Tochter Diener plasma ein, die Lohnbehandlung nach ISO 9001:2008 und 13485:2003 durchführt. „Steigende Auftragseingänge in der Lohnbehandlung sowie neue Plasmaanwendungen machen diesen Schritt notwendig“, sagt Christof Diener, Geschäftsführer von Diener. Zu den Plasmaanwendungen gehören das physi- kalische Reinigen durch den lonenbeschuss und, je nach Gasart, auch durch chemische Reaktionen, das Aktivieren von Kunststoff-Oberflächen durch Plasmabehandlung und das Ätzen. Seit dem 3. Quartal 2012 bietet Diener außerdem erstmals eine Vakuumanlage für die Lohnbeschichtung mit Parylene an. Bei Parylene handelt es sich um ein hydrophobes, optisch transparentes polymeres Beschichtungsmaterial, mit dem elektrische und elektronische Baugruppen vor Außeneinflüssen wie Staub, Korrosion, Dämpfen und Flüssigkeiten geschützt werden können.

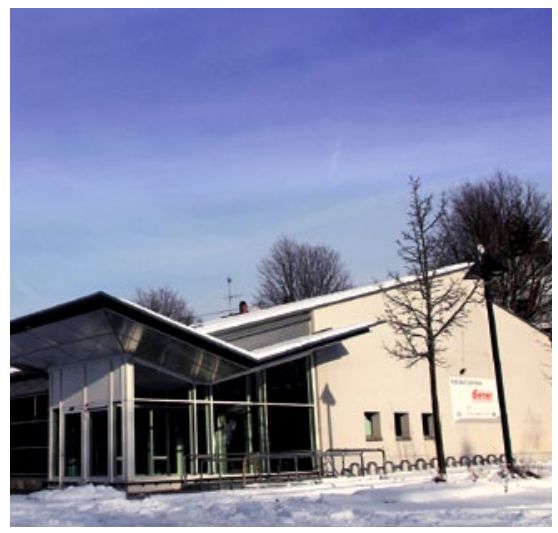

Diener expandiert und bezieht ganz in der Nähe der Firmenzentrale ein weiteres 845 Quadratmeter großes Gebäude. 